\title{
Crashes with roadside objects along motorcycle lanes in Malaysia
}

\begin{abstract}
Exclusive motorcycle lanes were introduced in Malaysia in the 1980s to reduce motorcyclist fatalities and they seem to be effective. However, no design guide has yet been developed for designing safer exclusive motorcycle lanes. The construction of Malaysian exclusive motorcycle lanes is based on the design criteria for cycle tracks [6], which does not suit the fast travelling and high volume of motorcycles along the exclusive lanes. According to the collected accident data, frequent motorcycle crashes still occur on exclusive motorcycle lanes. The present study found that roadside objects are one of the main contributing factors to motorcyclist fatalities. As a standard practice, guardrails have been planted along highways and roads. The design engineer of exclusive motorcycle lanes utilised the same practice and planted standard guardrails along exclusive motorcycle lanes. The suitability and the importance of the standard guardrail system as protection agent have been investigated and supported by real-world crash data. The catchment areas of the study were the top two longest exclusive motorcycle lanes in Malaysia: the exclusive motorcycle lanes along Federal Highway F0002 and the exclusive motorcycle lane along Shah Alam Expressway. Crash cases collected over 41/2-year period were from the accident database of the authorities. A total number of 107 cases were recorded as motorcycle crashes involving roadside objects. Guardrails have been identified as the most struck object, representing $32.7 \%$ of all roadside object-related motorcycle crashes along exclusive motorcycle lanes. An odds ratio analysis has found that narrow surface objects contribute to higher fatality rate than guardrails. However, guardrails still contribute $23.5 \%$ of all fatal roadside object-related crashes and were found to be 1.7 times more likely to cause serious injury to motorcyclists than nonobject-related motorcycle crashes. These findings support that guardrails are suitable to be used as a protection agent for the motorcyclists using the exclusive motorcycle lanes. However, further research and enhancements on the guardrail design system and material type are needed to have safer exclusive motorcycle lanes.
\end{abstract}

Keyword: Motorcycle crash; Motorcycle lane; Motorcycle-guardrail crash; Odd ratio analysis; Road sided object 